\title{
Multi-Layer Microbubbles by Microfluidics
}

\author{
Hongbo Zhang*, Haosu Meng, Qian Sun, Jianpu Liu, W. J. Zhang \\ Complex and Intelligent Research Centre, East China of Science and Technology, Shanghai, China \\ Email: *hbzhang@ecust.edu.cn
}

Received April 2013

\begin{abstract}
Multi-layer microbubble has great potential in enabling the corporation of medical imaging with tumor therapy such as drug and gene delivery of therapeutics or other functional materials in medical applications. Microfluidic technique has advanced over the last decade and showed great promise in replacing traditional microbubble generating method. In this paper, a multi-layer microbubble structure was produced with the aspect as potentially used for drug loaded microbubble contrast agents.
\end{abstract}

Keywords: Microfluidic; Multi-Layer; Microbubbles; Medical Imaging

\section{Introduction}

Microbubble as contrast agents in medical imaging is first invented in 1970s and it has greatly improved the quality of medical imaging over the decades. Recently, it has been found that microbubbles can be used to kill malignant cells by releasing preloaded drugs and also by enhancing the drug transfer efficiency in ultrasound filed. It can also assist the drug targeting to the cells by the micro-jetting emitted by the microbubble when it collapses subjected to an intense ultrasound filed. This concept has encouraged researches to generate microbubbles with unique structures that meet the demand, but so far, there are still numerous obstacles remained.

Microfluidic technique has been a promising tool to generate microbubbles owing to its number of merits, such as high monodispersity and controllable size distribution [1,2]. New attempts have been made to increase the production, for example, multi-array microchips [3] and sudden deepened configuration in the micro-channel [4]. The mechanism of microbubble generation by microfluidic is still not fully understood, but the most accepted theory is that in flow focusing (or co-flow) configuration, the microbubbles are generated by the instability of gasliquid jets. Therefore, it can produce much smaller microbubbles compared with the T-junction geometry [5]. The mechanism of the microbubble formation has been studied by the ultra high speed camera [6]. Their observation suggests that the final moment of microbubble pinch-off in a cross focusing system is purely liquid inertia driven; however, surface tension is still important.

The drug loaded microbubbles are mainly produced by attaching functional groups to the microbubble surface,

${ }^{*}$ Corresponding author. which suffers the major defect that only selects group of drugs can be loaded [7]. Multilayer microbubbles might hold the key to succeeding drug loading function with high efficiency. The drug or gene can be encapsulated in the middle layer with a protection outer layer in the shell. Also due to the immersion of the two layers, the outer layer can be liposome while the drug or gene aqueous liquid is in the middle. In this paper, a multi-channel microfluidic device was constructed to generate multi-layer microbubbles.

\section{Material}

The middle layer of the microbubble is between 80 (Amresco, Ltd.) and ionized water mixture $(2 \% \mathrm{w} / \mathrm{v})$, the outer layer is composed of liquid paraffin (Shanghai Lingfeng Ltd.) and air is chosen as the gas phase.

\section{Microbubble Generation}

The microfluidic configuration is shown in Figure 1. The depth of the channel is $25 \mu \mathrm{m}$, the width of the channel is $100 \mu \mathrm{m}$, the width of the joint is $20 \mu \mathrm{m}$ and the length of the channel for each input is $7 \mathrm{~mm}$.

\section{Experiments}

The experimental setup is shown in Figure 2. Compressed air was regulated by a valve (Swagelok B-SS4VI, USA) and a pressure sensor was used to monitor the pressure. Liquid phases were supplied by Syringe pumps (Stoelting 53100, USA). High speed camera (Microview MVC610DAM-GE110, USA) and microscopy (Sharpscope SF-1, USA) was used to evaluate the microbubble generation process and size distribution of microbubbles. 


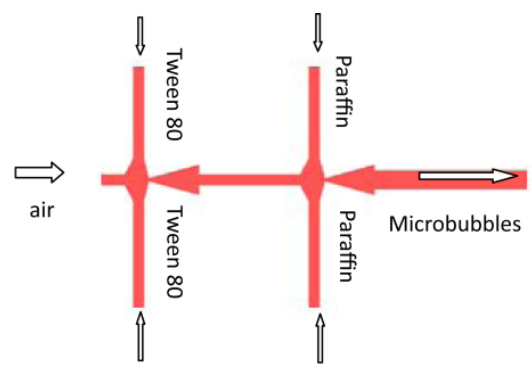

Figure 1. Microfluidic configuration.

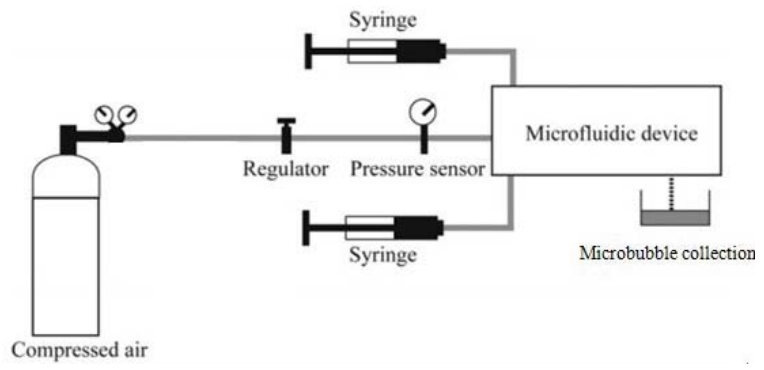

Figure 2. Experimental setup.

\section{Results and Analysis}

The air pressure was fixed at $23 \mathrm{psi}$, while the flow rate of the middle layer (tween 80 and water mixture) was set at $20 \mu \mathrm{l} / \mathrm{min}$, the flow rate of the outer layer of liquid paraffin varied between $5-15 \mu \mathrm{l} / \mathrm{min}$, multi-layer microbubbles were generated. Figure 3 shows the microbubble generating process. The microbubbles with much smaller size compared with the width of the channels were produced. Due to the secondary encapsulation, the final multi-layer microbubbles (as shown in Figure 4) have a relative larger size compared with monolayer microbubbles. The inserted photo clearly showed the multi-layer structure. The scale bar is $100 \mu \mathrm{m}$. The multi-layer microbubbles have a relative uniform size. The core of the microbubbles tends to move to the edge of the multi-layer structure, it is more likely because of the van der Waals force.

The flow ratio of two liquid phases plays an important role in determine the microbubble size and distribution when the gas phase pressure is fixed. At lower flow ratio of 0.25 and 0.4 , the microbubble production is low, at the ratio of 0.5 between 80 and liquid paraffin, the microbubbles size is becoming smaller as shown in Figure 5, with the increase of the both liquid phase flow rate, the microbubble becomes larger, but the dispersity of the microbubbles reduces (as shown in Figure 6). Due to higher flow rates, the process is becoming more stable. As the increase of the outer layer flow rate, the outer layer in the microbubble is becoming thicker as shown in Figure 4 compared with Figure 5. The scale bar in Figure 5 is $50 \mu \mathrm{m}$. It is possible to modify the membrane thickness of the microbubbles, therefore, it is likely to

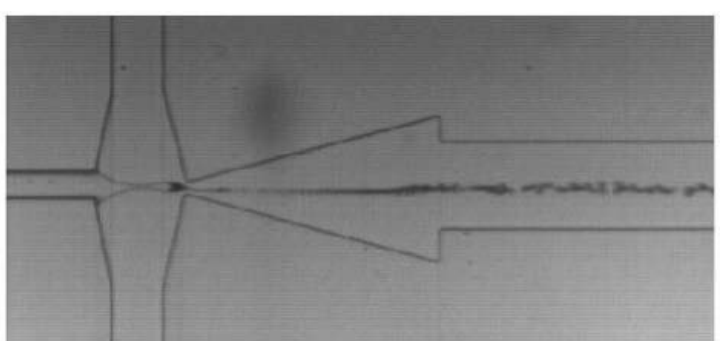

Figure 3. Microbubble generation.

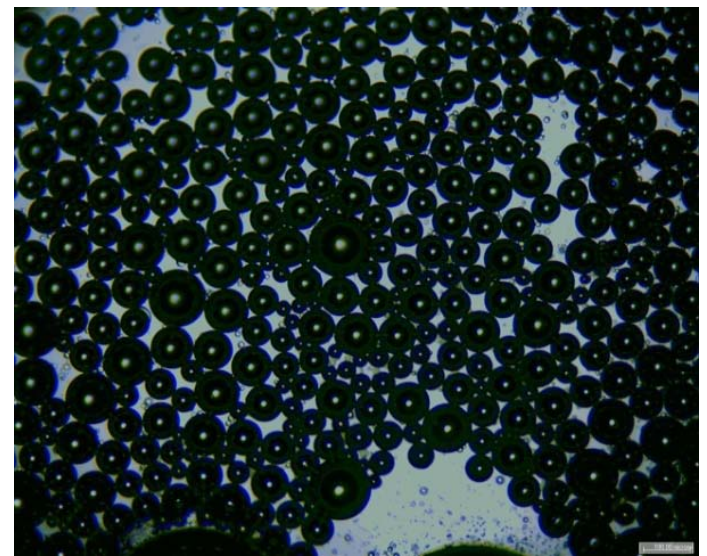

Figure 4. Multi-layer microbubbles (flow ratio of 0.75 ).

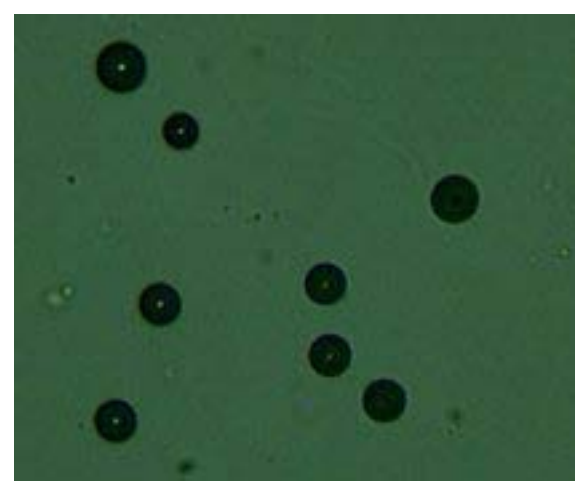

Figure 5. Microbubbles at liquid phase flow ratio of 0.5.

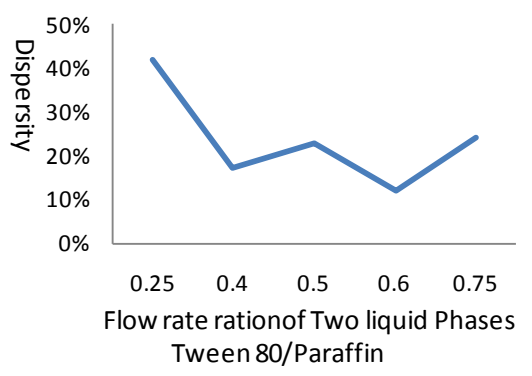

Figure 6. Microbubble dispersity.

adjust the amount of drugs and genes being carried by the microbubbles.

The dispersity index was calculated by the formula (1): 


$$
C V=\sigma_{d} / D_{m}
$$

where $\sigma_{d}$ is the standard derivation, and $D_{m}$ is the mean diameter of microbubbles.

Due to the higher surface tension of liquid paraffin to water of $72 \mathrm{dyn} / \mathrm{cm}$, the multi-layer microbubbles cannot survive for more than 10 minute. The properties of the immiscible two liquid could play an important role in prolonging the life time of microbubbles.

\section{Conclusion}

Multi-layer microbubble structures have been produced by microfluidics. By adjusting the operating parameters, such as flow rate ratio, the microbubble size can be varied, so as the thickness of the microbubble membrane and it could offer a potential mean for generation of drug and gene loaded microbubble contrast agents to accomplish the dual function as imaging and therapy of cancers.

\section{Acknowledgements}

This work is supported by the "One Thousand Talented Scheme" and the Fundamental Research Funds for the Central Universities in China.

\section{REFERENCES}

[1] E. Talu, K. Hettiarachchi, R. L. Powell, A. P. Lee, P. A. Dayton, and M. L. Longo, "Maintaining Monodispersity in a Microbubble Population Formed by Flow-Focusing," Langmuir, Vol. 24, 2008, pp. 1745-1749. http://dx.doi.org/10.1021/la703065v

[2] K. Y. Song, W. J. Zhang and M. M. Gupta, "Size-Controllable Monodispersed Microsphere Generation by Liquid Chopper Utilizing PZT Actuator," Journal of Microelectromechanical Systems, Vol. 22, No. 1, 2013, pp. 147151.

[3] M. R. Kendall, D. Bardin, R. Shih, P. A. Dayton and A. P. Lee, "Scaled-Up Production Using Multi-Array Microfluidic Module for Medical Imaging and Drug Delivery," Bubble Science, Engineering and Technology, Vol. 4, No. 1, 2012, pp. 12-20.

[4] S. A. Peyman, R. H. Abou-Saleh, J. R. Mclaughlan, N. Ingram, B. R. G. Johnson, et al., "Expanding 3D Geometry for Enhanced On-Chip Microbubble Production and Single Step Formation of Liposome Modified Microbubbles," Lab on Chip, Vol. 12, 2012, pp. 4544-4552. http://dx.doi.org/10.1039/c2lc40634a

[5] E. Castro-Hernandez, W. van Hoeve, D. Lohse and J. M. Gordillo, "Microbubble Generation in a Co-Flow Device Operated in a New Regime,” Lab on Chip, Vol. 11, 2011, pp. 2023-2029. http://dx.doi.org/10.1039/c0lc00731e

[6] W. Hoeve, B. Dollet, M. Versluis and D. Lohse, "Microbubble Formation and Pinch-Off Scaling Exponent in Flow Focusing Devices,” Physics of Fluids, Vol. 23, 2011, Article ID: 092001. http://dx.doi.org/10.1063/1.3631323

[7] I. Lentacker, B. G. Geest, R. E. broucke, et al., "Ultrasound-Responsive Polymer Coated Microbubbles That Bind and Protect DNA," Lamgmuir, Vol. 27, No. 17, 2006, pp. 341-334. 